\title{
Highly Flexible Feeding of Components for Automatic Assembly. The SyFA module
}

\author{
M.-O. Hongler, J. Jacot and Ph. Ney * \\ Département de Microtechnique \\ Institut de Production microtechnique \\ Ecole Polytechnique Fédérale de Lausanne \\ CH-1015 Lausanne
}

F. Arrieta, O. Ryser, Ch. Gertsch, J.-L. Lopez and F. Marques

NEW INGENIA

17, Route du Vignoble

CH-2017 Boudry

M. Frauenfelder and F. Walter

MIKRON

17, Route du Vignoble

CH-2017 Boudry

May 7, 2001

\begin{abstract}
A new highly flexible components feeder used in automatic assembly lines is presented. Flexibility is gained by the joint use of a vision system and a vibrating tooling plate which simultaneously acts as a separating and an orienting mechanism. A detailed mathematical modeling of the dynamics is derived. The modeling fully takes into account the random nature of the mechanisms which are used to extract out of a bulk of randomly oriented parts, those exhibiting a prescribed orientation.
\end{abstract}

*Supported by the "Commission Technologique à l'Innovation", (CTI). 


\section{Introduction}

Feeding parts, randomly oriented in a bulk phase, to a manipulator is one of the problem commonly encountered in automatic production lines. Despite to its conceptual simplicity, this problem often involves rather expensive solutions. The main reason responsible for these high costs is the relative lack of flexibility of most feeding devices. Indeed, to each particular types of parts, production engineers propose a world of fancy tricks and black magic which, due to their prototypic nature, always imply high costs, time consuming and perillous realizations. Since long, this difficulty has been recognized and has stimulated a sustained research and development activity. Among the pioneering work, we mention the studies of A.H. Redford et al. [Red 67] of G. Boothroyd et al. [Boo 82] in which a programmable vibrating feeder has been described. Since that early work, video equipment used in industrial contexts have been subject to tremendous progresses. Nowaday, several applications such as the positioning of components, the robot guidance, the quality control mechanisms, etc... commonly use vision systems. It is therefore not surprising that these techniques do also offer new solutions to the realization of part feeders. This is particularly true when the issue of flexibility is addressed. It is the aim of the present paper to describe a new approach to the part feeding problem which fully relies on the use of a vision equipment. Our development is focused on parts which typical sizes range from 0.5 to 5 [cm] with weight form 1 to 50 [grams]. Such components are commonly encountered in the shop floor. To gain flexibility, we reconsider the problem of part feeding from very basic considerations and we have taken as our main objective to suppress, as far as possible, the highly customized operations present in the classical feeders available on the market.

Our paper is organized as follows: in section 2, we present a brief review of the classical feeding devices in which we explicitly distinguish between the operations which are highly flexible and those which are specific to one particular type of component. This analysis is at the origin of the ideas which has guided the development of a new module, named SyFA (for System for Flexible Assembly) in the following. The SyFA module is registered under the European patent No. 99 810 403.8. The module is presented in Figures 2 for the prototypic realization and in Figure 2 for the operating principle. For this device, we need to describe the control parameters and to define the operating policy which regulate the flow rate of oriented components. We propose in sections 3 to 6 a detailed mathematical modeling of the dynamics of the SyFA device. In section 3, the approach towards a 
statistical equilibrium for an assembly of identical components on a vibrating tooling plate is discussed. In section 4, the image processing policy adopted is shortly described. In section 5 , the maximal output flow rate is estimated. We pay a special attention on the fluctuations around the average performances as they partly characterize the reliability of our device. Finally in section 6 , the reader will find a conclusion.

\section{$2 \quad$ Flexibility in feeding devices and the SyFa concept}

In their classical versions, parts feeders exhibit generically the following series of operations:

a) From a bulk of randomly oriented parts, a vibrating bowl, a conveying track or any other conveying device, generates a linear flow of separated components resting in random orientations. Let $\mathcal{D}$ be the output flow rate of this randomly oriented components.

b) The linear flow of randomly oriented components is driven through a series of orienting and selecting devices. After these devices, a linear flow of correctly oriented parts emerges. It obviously has, due to the selection mechanisms, a reduced flow rate $\mathcal{D}^{\prime} \leq \mathcal{D}$. The components with the "wrong" orientations are returned to the bulk of random parts.

c) Finally each correctly oriented component is localized in a referential system that can be used by the robot. The robot then extracts the component from the feeder and proceeds to the assembly operation.

Clearly the selection devices and part of the orienting devices offer a rather poor degree of flexibility. Indeed, any change in the size and/or the geometry of components to be fed implies the re-design of costly hardware. Observe nevertheless that among the large variety of orienting devices, the simple jump of components over a simple step with a selected height is almost ubiquitous in actual realizations, [Boo 82]. The simplicity of this jump processes offers a high degree of flexibility, (i.e. the modification of the height of a step is essentially free of any cost).

In the SyFA module only the jump mechanism is retained. The SyFA operates as follows, (see also Figure 2): a collection of components with random orientation is extracted from the bulk of randomly oriented parts and dropped on a tooling plate which is intermittently 
set into vibration. During the vibrating phases, the components which crosses the free edge of the tooling plate are recycled into the bulk. Two essential functions are simultaneously realized during a vibrating phase, namely a) the separation of components and b) the changes of orientations of the components due to their vibro-impact motions over the tooling plate. At the end of the vibration phase, which duration has to be adjusted, the tooling plate is resting and a vision system is able to localize and recognize the components exhibiting the required orientation. Finally a robot extract the correctly oriented components from the tooling plate. Then a new vibrating phase is activated and the procedure is iterated until the number of components left on the vibrating plate reaches a minimal level $N_{i n f}$. When $N_{i n f}$ is crossed, a refilling mechanism of the vibrating plate is triggered. Clearly the vibro-impact jumps and the image processing processes characterizing the SyFA device offer a high degree of flexibility. In this manner, we have clearly suppressed the need to generate a linear flow of separated and correctly oriented components which is highly customized in the classical feeders. Note that the feeder recently described in [Wol 97], does also use a vision system but it retain the needs to generate a linear flow of (in this case randomly oriented), separated parts.

The basic sequence of operations of the system forms a renewal cycle which is sketched in Figure 2. The renewal cycle length is a random time $\tau_{n}, n=1,2, \ldots$, which can be decomposed into the following typical sequence of phases see Figure 2:

1) time interval $\left[\theta_{j}, \theta_{j+1}\right]$ : Lift of the feeding cone and drop $N_{0}$ components at random on the tooling plate (TP). We shall refer to it as the refilling phase.

2) time interval $\left[\theta_{j+1}, \theta_{j+2}=\theta_{j+1}+z\right]$ : Vibration phase during a time interval of length $z$ [sec]. We shall refer to it as the vibrating phase.

3) time interval $\left[\theta_{j+2}, \theta_{j+3}=\theta_{j+2}+\tau_{v i s i o n}\right]$ : During the time interval $\tau_{\text {vision }}$, the vision system takes, stores and processes an image. We shall refer to it as the imaging phase.

4) time interval $\left[\theta_{j+3}, \theta_{j+4}=\theta_{j+3}+\tau_{\text {extr }}\right]$ : Extract component with the required orientation. We shall refer to it as the extracting phase. The time $\tau_{\text {extr }}$ ] characterizes the time needed to extract and assemble a correctly oriented component.

5) time interval $\left[\theta_{j+4}, \theta_{j+5}=\theta_{j+4}+z\right]$ : Second vibration phase of duration $z$ [sec].

- ...

- ... 


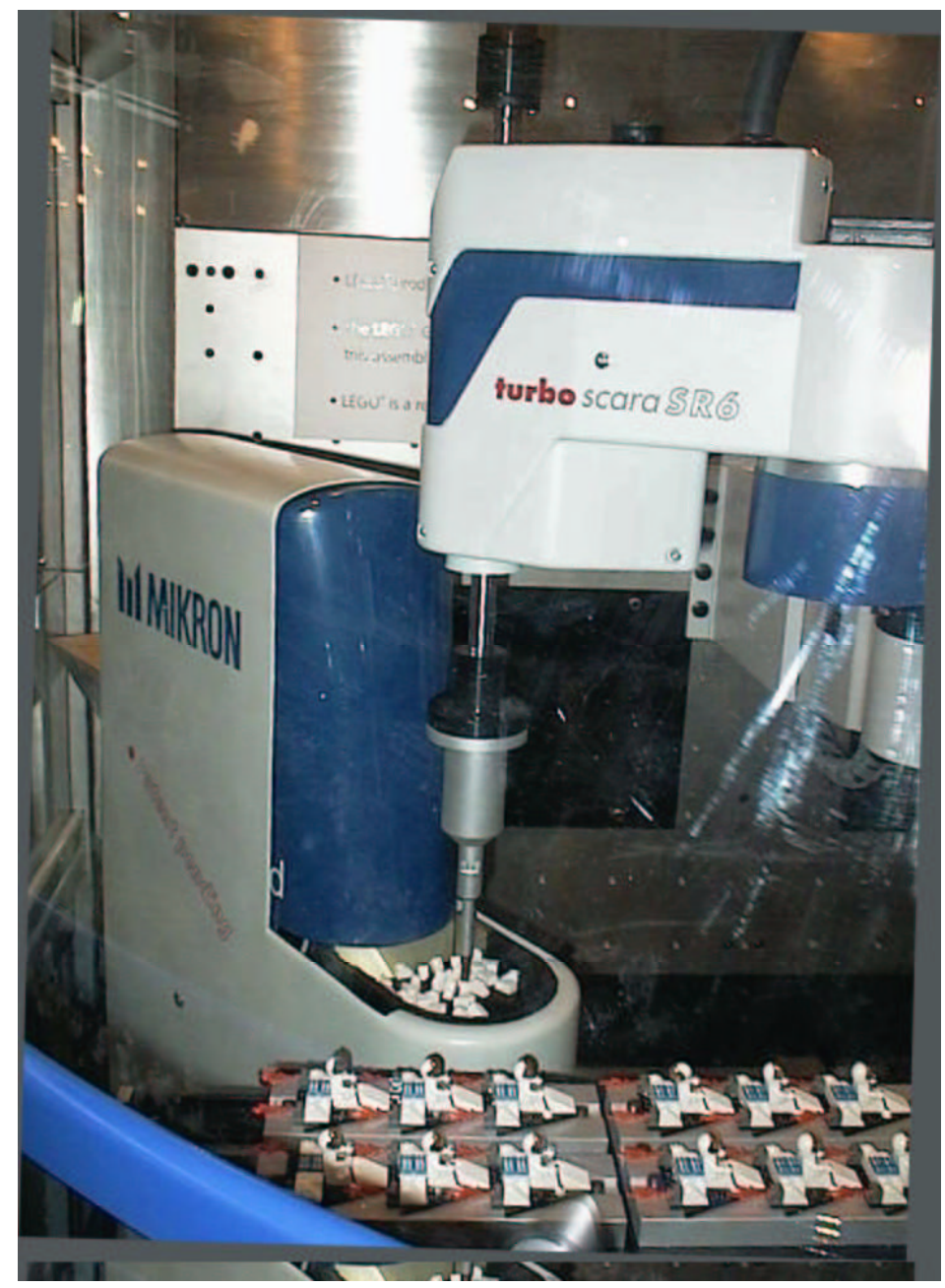

Figure 1: Prototypic realization of the SyFA module.

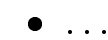

$\bullet \ldots$

M) time $\tau_{j}$ : End of the $j$-th renewal cycle.

The end of the cycle occurs after $M$ extraction and vibration phases. It is triggered when the number of components remaining on the tooling plate is smaller or equal to $N_{i n f}$. We observe that the process starts anew each time the refilling phase of the tooling plate is triggered.

Accordingly, the operation of the feeders depends on the following external control parameters:

a) The length of a vibration phase $z$ [sec] 


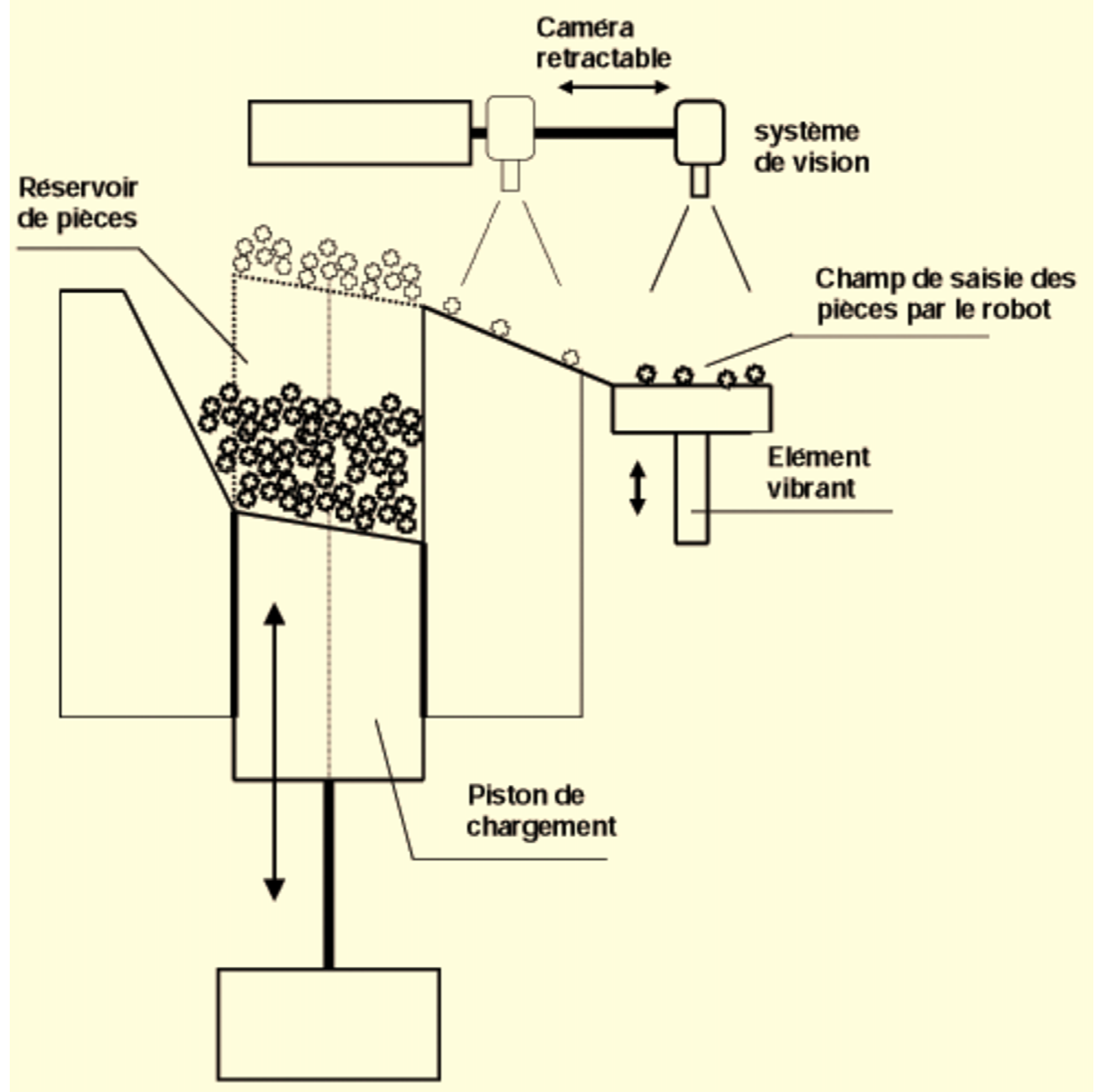

Figure 2: Operating scheme of the SyFA module.

b) The amplitude of the vibration $\rho_{s}[\mathrm{~m}]$, (i.e. $\rho_{s}$ is in fact the dominant Fourier mode of the vibration).

c) The frequency of the vibration $\omega_{s}=2 \pi \nu_{s}\left[\frac{1}{\mathrm{sec}}\right]$

d) $N_{0}$ the number of components dropped on the tooling plate just after refilling and prior to the first vibration phase.

e) $N_{i n f}$ the limiting population of components on the tooling plate before the refilling is ordered.

Note that at the beginning of the renewal cycle, the system always starts with a vibrating phase in order to separate the components freshly fed on the tooling plate. 


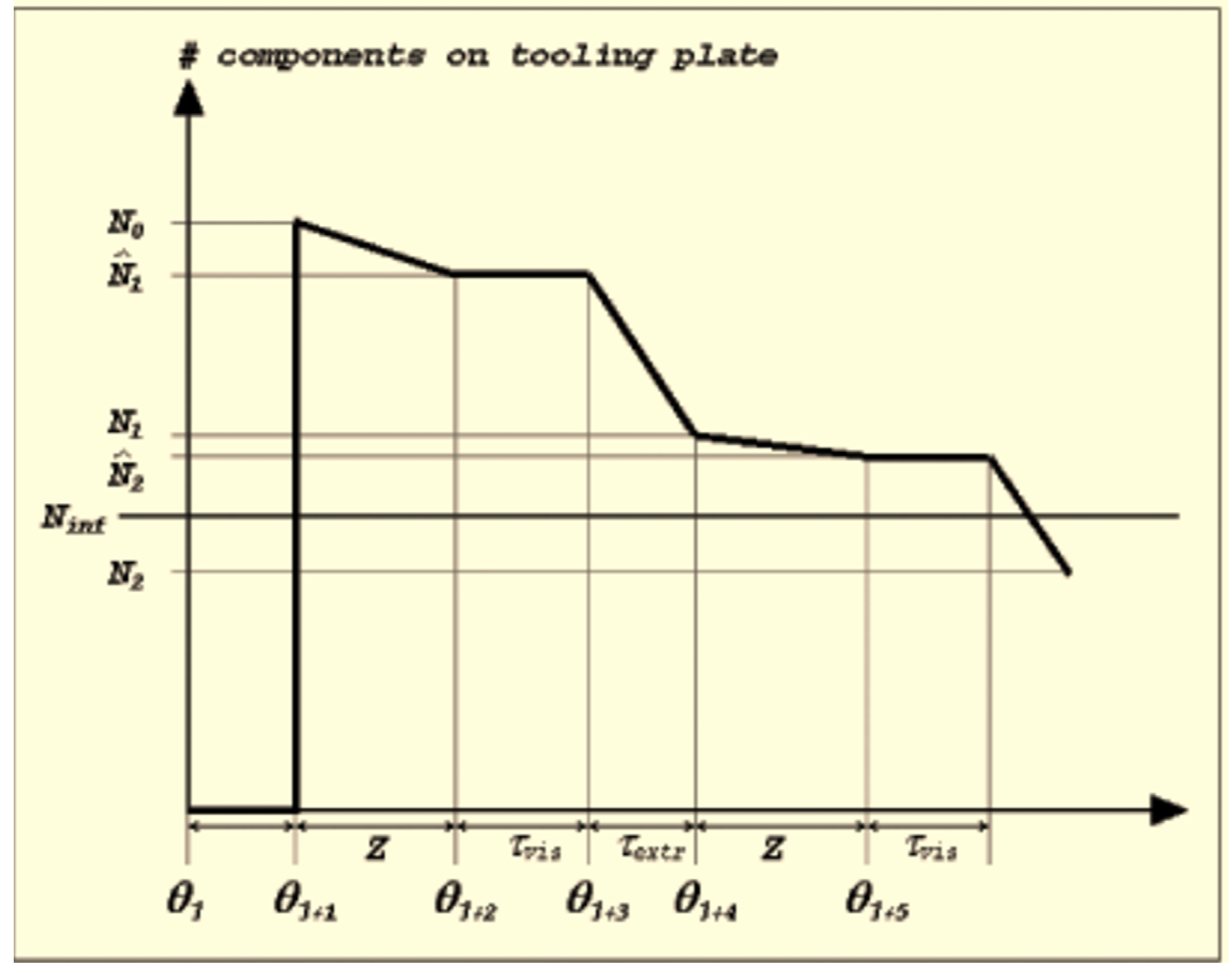

Figure 3: Sketch of a renewal cycle $\tau_{j}$. Here the renewal cycle stops after two vibrating phases, hence $M=2$.

\section{How to reach a statistical equilib- rium for an assembly of components}

Consider a collection of $N_{0}$ identical parts freshly fed on the tooling plate. After the first vibrating phase, there remain $\hat{N}_{1}$ components on the tooling plate. We assume that these components are separated. They sit on one among the $e_{1}, e_{2}, \ldots, e_{K}$ stable resting positions. We shall say that the assembly is in the statistical equilibrium if we approximately observe: $\hat{N}_{1} p_{1} \simeq \hat{n}_{1,1}$ parts in the position $e_{1}, \hat{N}_{1} p_{2} \simeq \hat{n}_{2,1}$ parts in the position $e_{2}, \ldots, \hat{N}_{1} p_{K} \simeq \hat{n}_{K, 1}$ parts in the position $e_{K}$, where $p_{1}, p_{2}, \ldots p_{K}$ are the natural equilibrium resting probabilities associated with the component under study. Note that strict equalities in the preceding expressions are obtained only in the limit $\hat{N}_{1} \rightarrow \infty$. 
Roughly speaking, when randomly throwing a component onto the tooling plate, it will rest with probabilities $p_{1}, p_{2}, \ldots p_{K}$ on the respective positions $e_{1}, e_{2}, \ldots, e_{K}$. The probabilities $p_{1}, p_{2}, \ldots p_{K}$ can be determined either experimentally or by using the theory exposed in [Lee 97] which is based on geometric considerations. As an illustration, for the part described in Fig. 4 below, we have measured after 100 trials $p_{1} \simeq 0.28, p_{2} \simeq 0.03, p_{3} \simeq 0.55, p_{4} \simeq 0.1, p_{5} \simeq 0.03$ and $p_{6} \simeq 0.01$. In this application, the required orientation is $e_{3}$ with probability $p_{3} \simeq 0.55$.

For simplicity in the notations, we shall assume in the following, without loss of generality, that $e_{1}$ is the required orientation. Consider now the situation which occurs when, after the first extraction phase. The remaining collection of parts is composed of $N_{1} \leq \hat{N}_{1}$ entities. This last assembly is drawn at random from an off-equilibrium distribution characterized by:

$$
n_{1,1}=0 \Rightarrow n_{2,1} \neq N_{1} p_{2}, \ldots n_{K, 1} \neq N_{1} p_{K}
$$

with $\sum_{j=1}^{K} n_{j, 1}=N_{1}$. Starting now a new vibrating phase of duration $z$ [sec], the population of parts remaining on the tooling plate will be denoted by $\hat{N}_{2}$. Two questions can now be raised:

a) Do we approach the statistical equilibrium distribution ? In other words do we observe, after the vibrating phase of length $z$ [sec], a configuration such that:

$$
\hat{n}_{1,2} \simeq \hat{N}_{2} p_{1}, \hat{n}_{2,2} \simeq \hat{N}_{2} p_{2}, \ldots, \hat{n}_{K, 2} \simeq \hat{N}_{2} p_{K}
$$

b) If the answer to a) is affirmative, how to select $z$ in order to reach the equilibrium given by Eq.(2)?

\subsection{Tooling plate excitation needed to drive a population of components towards its statisti- cal equilibrium}

To answer question a), we examine the action of the vibrating tooling plate on a assembly of components. First, we introduce the minimal potential barrier $\Delta W_{k, j}$ that has to be overcome for a component starting in the position $e_{k}$ to end, after perturbation, in the new resting position $e_{j}$. We can write:

$$
\Delta W_{k, j}=m g \Delta h_{k, j}
$$

where $m$ is the mass of the part, $g=9.81\left[\frac{\mathrm{m}}{\mathrm{sec}^{2}}\right]$ is the module of the gravitation acceleration and $\Delta h_{k, j}$ is the minimal height difference of 
the centroid needed to transit from position $e_{k}$ to $e_{j}$. To illustrate this last concept, consider a cubic dice with edge $a$. To transit from one face to another adjacent face, we have $\Delta h_{k, j}=\frac{a}{2}(\sqrt{2}-1)$ and $\Delta h_{k, j}=\frac{a}{2}(\sqrt{3}-1)$ for non-adjacent faces. Clearly, to reach statistical equilibrium, hence to be able to explore all the potentially resting positions, the vibrating plate has to lift up the components at an effective height $h_{e f f}$ larger than $\max _{k, j} \Delta h_{k, g}$.

To give some quantitative estimations, let us now examine the dynamics of a bouncing ball on a vibrating table, (i.e. here the tooling plate). At each impact with the table, energy is dissipated as the impact is quasi-plastic. Even for this elementary situation, which belongs to the class of vibro-impact dynamics, unexpectedly complex behavior, (i.e. cascades of period doubling bifurcations and chaotic orbits) result, (see [Guck 83] and [Hong 94] for a detailed analysis).

Assume that the tooling plate follows a periodic motion described by a function $f(t)=f(t+T)$. By Fourier analyzing, we can write :

$$
f(t)=\sum_{j \geq 1} \rho_{j} \sin \left(\omega_{j} t\right) .
$$

For simplicity and in accord with actual realizations, we shall further assume that a single mode $j=s$ of angular velocity $\omega_{s}$ and amplitude $\rho_{s}$ dominantly contributes to the excitation force $f(t)$. Hence, in the reference frame moving with the tooling plate, the equation of the motion for a component of mass $m$ resting on the tooling plate at time $t=0$ reads:

$$
m \frac{d^{2}}{d t^{2}}\{X(t)+f(t)\}=-m g, \quad X(t) \geq 0,
$$

where $X(t)$ stands for the position in the vertical direction above the tooling plate. From Eq.(5), we can approximately write:

$$
m \frac{d^{2}}{d t^{2}} X(t) \simeq-m g+m \rho_{s} \omega_{s}^{2} \sin \left(\omega_{s} t\right)
$$

Hence the minimal acceleration for the component to leave the tooling plate immediately reads:

$$
\rho_{s} \omega_{s}^{2}>g
$$

Clearly Eq.(7) furnishes a lower bound for the excitation necessary for an approach to statistical equilibrium. Note however that Eq.(7) is not a sufficient condition. 


\subsection{The orientation process generated by a succession of jumps}

The approach toward the statistical equilibrium using orienting devices has been discussed in [Seth 90], [Jaum 90] and [Hong 94]. Accordingly, we give here only a short and simplified review of this problem sufficient for our present needs. We shall assume that $\left\{e_{1}\right\}=H$, (we write $H$ for head), is the required orientation and we shall lump all other stable resting positions into a single state say $T=\left\{e_{2}, e_{3} \ldots e_{K}\right\}$, ( $T$ for tail). The behavior of the orienting mechanism will be described in this reduced state space $\{H, T\}$. Hence, when the statistical equilibrium is reached the associated probabilities are $p_{H}=p_{1}$ and $p_{T}=\left(1-p_{H}\right)=\sum_{j=2}^{K} p_{j}$ respectively. After extraction by the robot, the components remaining on the tooling plate are in an offequilibrium distribution characterized by:

$$
\widetilde{p_{H}}=0 \text { and therefore } \widetilde{p_{T}}=\left(1-\widetilde{p_{H}}\right)=1 .
$$

Eq.(8) simply states that no component remains in the required orientation after the extraction phase. Now, during the vibration phase, each time the components has an impact on the tooling plate, its orientations is changed and therefore the off-equilibrium statistical distribution given by Eq.(8) is also modified. We represent the change on the initial distribution Eq.(8) obtained after a single impact by the action of a matrix operator $\mathbf{T}$, namely:

$$
(0,1) \mathbf{T}=\left(p_{H, 1}, p_{T, 1}\right)=(\gamma, 1-\gamma),
$$

where the transition matrix $\mathbf{T}$ is defined as:

$$
\mathbf{T}=\left(\begin{array}{cc}
(1-\beta) & \beta \\
\gamma & (1-\gamma)
\end{array}\right)
$$

with $(1-\beta)$ denotes the probability that a component which is in the correct orientation before a jump stays in this correct orientation after the jump. Hence, $\beta$ is the probability that a part initially with a correct orientation is left, after the jump, with the wrong orientation. Finally $\gamma$ is the probability that a part with a wrong orientation initially is left in the correct orientation after the jump and $(1-\gamma)$ is the probability that a component initially in the wrong orientation remains in this wrong orientations after a jump.

Assuming that the effects of successive impacts produce identical statistical effects, we can write after $n_{i m p}$ impacts, (see [Hong 94] for the details of the calculations): 
$(0,1) \mathbf{T}^{n_{i m p}}=\left(p_{H, n_{i m p}}, p_{T, n_{i m p}}\right)=\left(\frac{\gamma}{\beta+\gamma}-\delta\left(n_{i m p}\right), \frac{\beta}{\beta+\gamma}+\delta\left(n_{i m p}\right)\right)$,

with:

$$
\delta\left(n_{i m p}\right)=\gamma \frac{(1-\beta-\gamma)^{n_{i m p}}}{\beta+\gamma}
$$

For $z$ large enough, the number of impacts $n_{i m p}$ will be high and as $|1-\beta-\gamma| \leq 1$ we shall reach:

$$
\lim _{n_{i m p} \rightarrow \infty}(0,1) \mathbf{T}^{n_{j u m p}}=\left(\frac{\gamma}{(\beta+\gamma)}, \frac{\beta}{(\beta+\gamma)}\right)=\left(p_{H}, 1-p_{H}\right)
$$

A sketch of two typical situations, depending on whether $1-\beta-\gamma$ is positive or negative, is given in Figures $4 \mathrm{a}$ ) and $4 \mathrm{~b}$ ).

Note that while in the case Fig. 4a) for which $1-\beta-\gamma>0$, the approach towards the equilibrium point in monotonous, this is not true for case Figure $4 \mathrm{~b}$ ) where $1-\beta-\gamma \leq 0$; here the approach is alternating. Hence, we can conclude that:

a) $1-\beta-\gamma>0$. Here, the more impacts we have, the higher the probability to observe the correct orientation will be. This indicates that large vibrating times $z$ implying a large $n_{i m p}$ are in favor. In actual applications, 5 to 10 impacts are already sufficient to approach the equilibrium value given in Eq.(13), (see also figure 4a). As the frequency of the tooling plate $\nu_{s}$ is of the order of $20[\mathrm{~Hz}]$, it is clear that a vibrating time of the order $z=1$ [sec], which implies $n_{\text {imp }}$ to be of the order of 10 to 20 , will drive the statistical assembly near its equilibrium.

b) $1-\beta-\gamma \leq 0$. In this case, a single impact will be required to have the higher probability to observe the correct orientation. In practise, this will however hardly be realized. When this situation is encountered, a short vibration phase $z$ is sufficient.

\section{Image Processing}

After a vibrating phase, the vision system first grabs an image with 256 grey levels and operates a binarization of the picture. This operation is performed with an ad-hoc threshold level which depends on the components under study, the light source used and other specific features. The vision system then uses this binarized picture to select the components exhibiting the correct orientation. The filtering process is performed via two phases, namely the "blob" analysis and the 


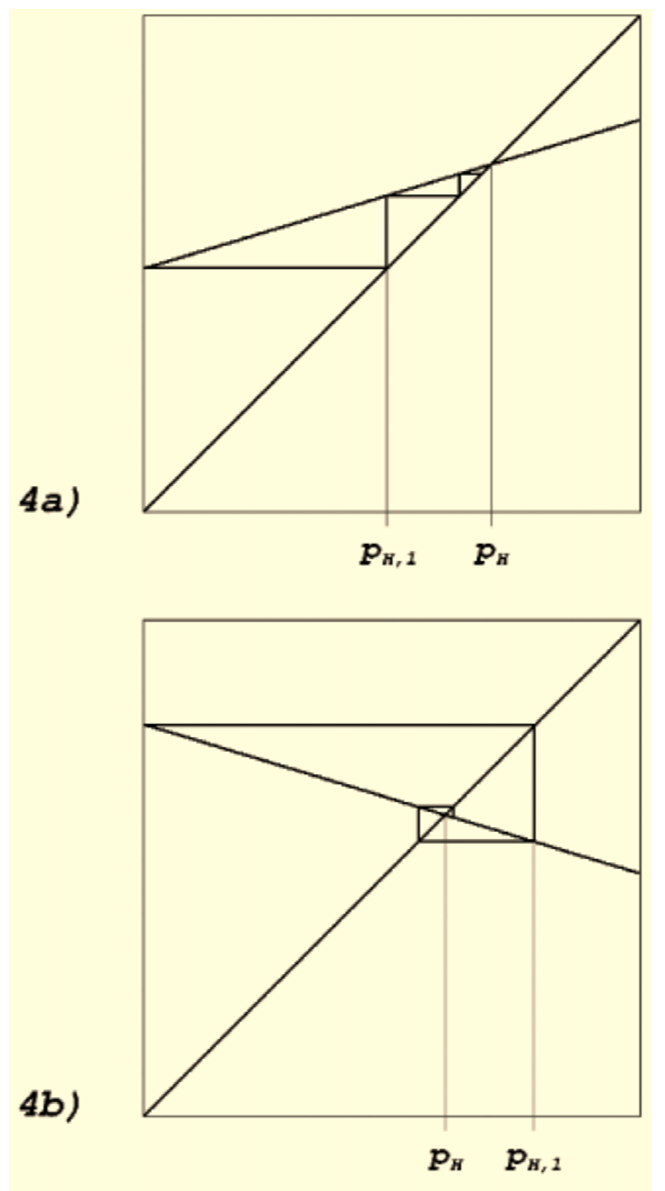

Figure 4: Approach to equilibrium via successive jumps. In the case a) $0<1-\beta-\gamma<1$ while in the case b), we have $-1<1-\beta-\gamma<0$

"pattern matching". The basic operations performed by these filters can be summarized as follows:

a) Blob analysis

Determination from the binarized image of the basic geometric parameters, (area, perimeter, gravity center, principal axes of symmetry,...) of the spots forming the binarized picture.

b) Pattern matching

Localization in selected portions of the grey level source image, (i.e. the grabbed image), of a predefined pattern. The relevant portions of the source image are themselves determined by the blob analysis. 
The blob analysis is systematically applied by the SyFA module. It is a very rapid operation for which the typical processing time ranges around $\tau_{0} \simeq 0.15$ [sec]. In simple applications, the blob analysis is already sufficient to both select the correctly oriented components and determine the relevant coordinates to be used by the robot, (see Figure 4 for an example where the "blob analysis is sufficient).
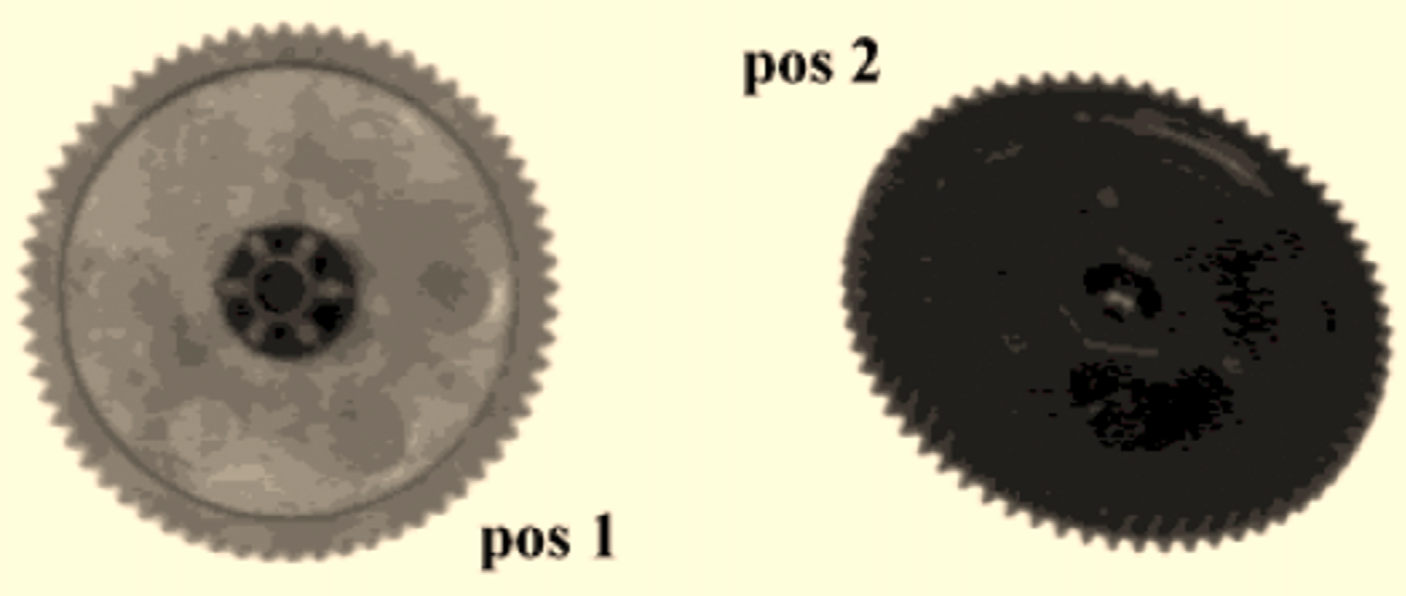

Figure 5: Example of a component, a plastic cog wheel, which requires only the blob analysis

For more complex cases, with for instance left-right symmetries and other more "refined" geometric structures, the blob filters cannot alone yield the required information. For these cases, the vision system uses the pattern matching analysis to lift the unresolved indeterminacies. Note that the pattern matching filter is more time consuming: the processing time is proportional to the number of components detected on a picture. Typical times are here of the order of $s=0.3\left[\frac{\mathrm{sec}}{\mathrm{pce}}\right]$. In Figure 4, an example of a component which require the pattern matching filter is exhibited.

\section{Modeling of the flow of oriented com- ponents}

Let us now come to the mathematical modeling of the dynamics of the SyFA feeder. We denote by $\mathcal{D}(M, z) \frac{[\mathrm{part}]}{[\mathrm{sec}]}$ the output rate of oriented parts delivered by the robot during one renewal cycle. Remember that the parameters $M$ and $z$ are respectively the number of vibration 


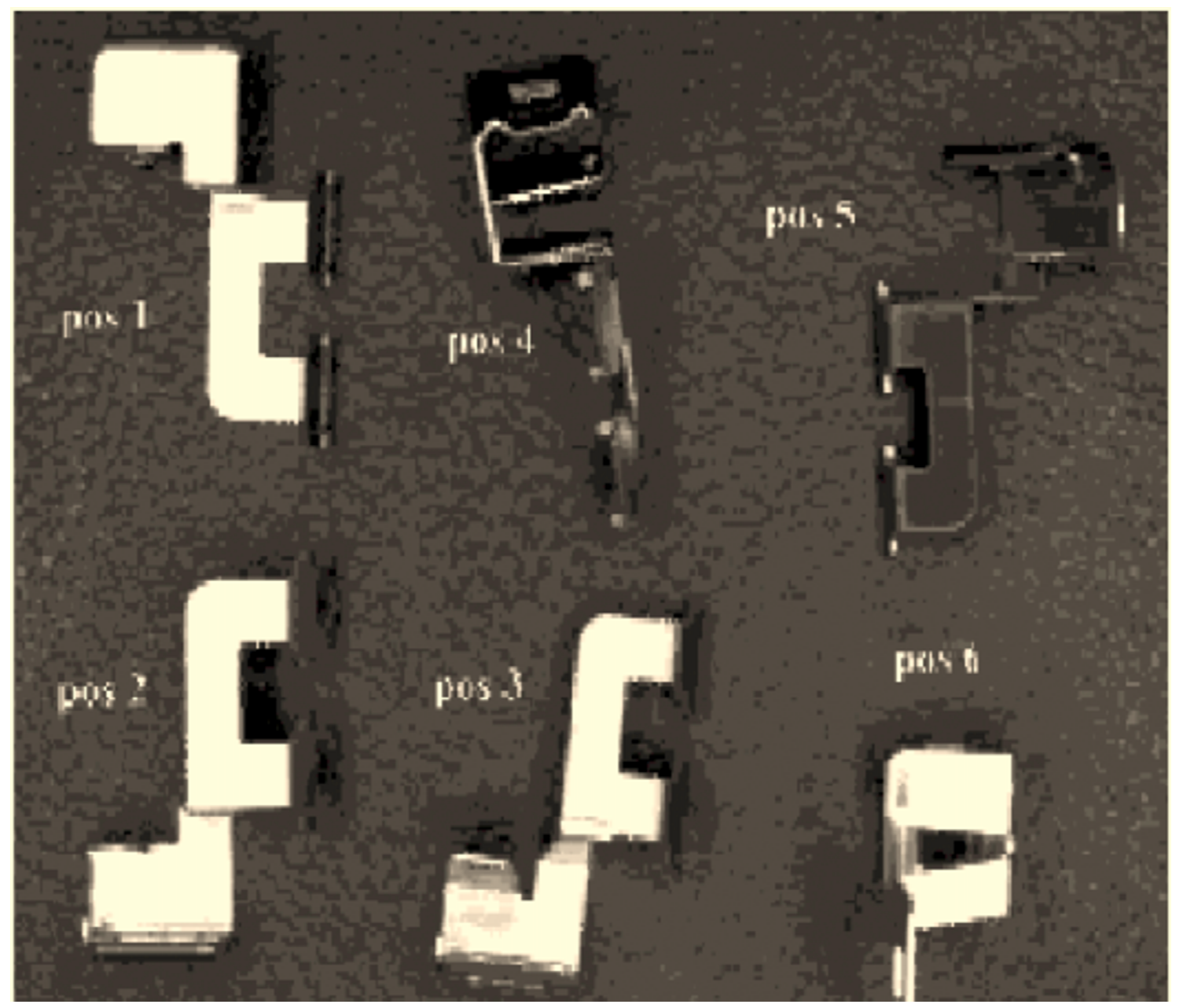

Figure 6: Example of a component, a copper connector, for which the pattern matching analysis is mandatory.

phases in a renewal cycle and the duration of a single vibration phase. Now, we can formally write:

$$
\mathcal{D}(M, z)=\frac{\mathcal{R}(M, z)}{u+M\left(\tau_{0}+z\right)+\mathcal{R}(M, z)(v+s)},
$$

where we have introduced the following new definitions:

- $\mathcal{R}(M, z)$ [part]. Total yield of the correctly oriented components during one complete renewal cycle.

- $\tau_{0}$ [sec]. Systematic time needed for the image acquisition and to perform the blob analysis. In our device, we typically measure $\tau_{0}=0.15[\mathrm{sec}]$ 
- $u$ [sec]. Time needed to lift the feeding jack. In the SyFA module this time is approximately 0.5 [sec].

- $v \frac{[\mathrm{sec}]}{[\mathrm{part}]}$. Processing time of image to uncover a single well oriented component when pattern matching is activated.

- $s \frac{[\mathrm{sec}]}{[\text { part }]}$. Time needed to pick one component after it has been recognized to be in the ad-hoc orientation. A lower bound for this time interval is 0.5 [sec]. Usually this time will be of the order of 2 to 3 [sec].

Note that both $\mathcal{D}(M, z)$ and $\mathcal{R}(M, z)$ are both random variables. To calculate the yield $\mathcal{R}(M, z)$, we make the following hypotheses:

H1) During the vibrating phase, part of the components are driven out of the tooling plate. They are recycled into the bulk random phase. Let us write $\alpha(z) \in[0,1]$ the ratio of the components left on the tooling plate after a vibrating phase of time length $z$. We clearly have:

$$
\alpha(z=0)=1 \quad \text { and } \quad \alpha(z=\infty)=0 .
$$

The function $\alpha(z)$ is monotonously decreasing. We shall phenomenologically model it by:

$$
\alpha(z)=\exp \left\{-\lambda\left(\rho_{s}, \omega_{s}\right) z\right\},
$$

where the the parameter $\lambda\left(\rho_{s}, \omega_{s}\right)$ depends on the strength and on the frequency of the vibration of the tooling plate. It is easy to experimentally measure $\lambda\left(\rho_{s}, \omega_{s}\right)$. Using the SyFA module, several experimentations involving different components, have been performed. It is found that the law Eq.(16) holds asymptotically in $z$. For short $z$ however, discrepancies have been observed. These can be explained by the fact that a large number of components are present near the edge of the tooling plate just after its refilling by the pneumatic jack. Hence, at the beginning of the renewal cycle, the population lost of components from the tooling plate is larger. After this short initial phase, the lost regime follows the exponential law given in Eq.(16). For the components described in Fig 4, we found an approximate value $\lambda \simeq 0.12$ for $\nu_{s} \simeq 20[\mathrm{~Hz}]$ and $\rho_{s}=0.001[\mathrm{~m}]$. Note that for this choice of the excitation parameters, we have: $\rho_{s} \omega_{s}^{2}=\rho_{s}\left(2 \pi \nu_{s}\right)^{2}=15.8>9.81 \frac{[\mathrm{m}]}{[\mathrm{sec}]^{2}}$.

H2) After an extraction phase, the probability to have a component left in the correct orientation is assumed to be zero, (i.e. all correctly oriented parts have been extracted). For a vibration 
phase of duration $z$ [sec], we have a probability $p(z)$ to observe a component in the required orientation. Hence, we can write:

$$
p(z=0)=0 \quad \text { and } \quad \lim _{z \rightarrow \infty} p(z)=p_{H} .
$$

The rate of approach towards the statistical equilibrium has been described in section 3.2 and the time interval $z$ should be chosen accordingly.

We calculate the yield $\mathcal{R}(M, z)$ by assuming that the feeding jack has dropped $N_{0}$ components on the tooling plate. After a vibrating phase of $z$ [sec], the plate remains populated with $N_{0} \alpha(z)=\hat{N}_{1}$ components. Among these $N_{0} \alpha(z)$ components, we shall have $N_{0} \alpha(z) p(z)$ components with the required orientation, these will be evacuated by the robot. Accordingly, $N_{0} \alpha(z)(1-p(z))=N_{1}$ components will enter into the second vibrating phase, (see also Figure 2. Hence, $N_{0} \alpha^{2}(z)(1-p(z)) p(z)$ components will present the required orientation at the end of the vibrating phase. By successive iterations, we can write:

$$
\begin{aligned}
& \mathcal{R}(M, z)=N_{0} \alpha(z) p(z)+N_{0} \alpha^{2}(z)(1-p(z) p(z)+\ldots \\
& \ldots+N_{0} \alpha^{M+1}(z)(1-p(z))^{M} p(z)= \\
& =N_{0} p(z) \alpha(z) \sum_{j=1}^{M} \alpha(z)(1-p(z))^{j-1}= \\
& =N_{0} p(z) \alpha(z) \frac{1-[\alpha(z)(1-p(z))]^{M}}{1-\alpha(z)(1-p(z))}, \quad M \geq 1 .
\end{aligned}
$$

Observe that the behavior of Eq.(18) is in agreement with intuition. We indeed have:

- For $M>>1$ and $\alpha(z) \approx 1$, we obtain that $\lim _{M \rightarrow \infty} \mathcal{R}(M, 1)=$ $N_{0}$.

- For $\mathrm{M}=1$, we have $\mathcal{R}(1, \alpha(z))=N_{0} p(z) \alpha(z)$.

Alternatively, elementary considerations enable to express explicitly the total number $\mathcal{E}(M, z)$ of parts ejected from the tooling plate after $M$ vibrating phases. We obtain:

$$
\mathcal{E}(M, z)=N_{0}(1-\alpha(z)) \frac{1-[\alpha(z)(1-p(z))]^{M}}{1-\alpha(z)(1-p(z))}
$$

and finally, the total number of components $N(M, z)$ lying on the tooling plate after $M$ vibrating and extraction phases reads as:

$$
N(M, z)=N_{0} \alpha(z)^{M}(1-p(z))^{M} .
$$


From Eqs.(18), (19) and (20), we can verify the balance:

$$
\mathcal{R}(M, z)+\mathcal{E}(M, z)+N(M, z)=N_{0}, \quad \forall M \geq 1 .
$$

\subsection{Determination of $N_{i n f}$}

Remember from section 2 and Figure 2 that when the population of the parts on the tooling plate is smaller than $N_{i n f}$, a new cycle is initiated. From the definition of $N(M, z)$ given in Eq.(20), we can write:

$$
N(M, z) \leq N_{\text {inf }}
$$

On the other hand, $N_{i n f}$ should not be too small to prevent situations where a vibrating phase yields no correctly oriented component. Hence, the estimation of a safe value of the level $N_{i n f}$ is important. It will necessarily include probability considerations based not only on averages but also on variances. To estimate $N_{\text {inf }}$, let us consider the following model. As before in section 3.3, we group the "wrong" resting positions of the component into a single state $T=\left\{e_{2}, e_{3}, \ldots e_{n}\right\}$ and the correct orientation is $H=\left\{e_{1}\right\}$. Assume that the time length $z$ is selected to approximately ensure $p(z) \simeq p_{H}$. To each vibrating phase, we may idealize our process as being a Bernoulli trial composed of several tokens $\{H, T\}$. After a vibrating phase and prior to extraction, assume that we have $\hat{N}$ parts on the tooling plate. According to the Bernoulli distribution, the average number of parts with the right orientation will be approximately given by $\langle\hat{n}\rangle=\hat{N} p_{H}$ and its variance $\sigma_{H}^{2}$ will be $\sigma_{H}^{2}=\hat{N} p_{H}\left(1-p_{H}\right)$, [Mey 75]. Let us write $Q(\eta)=\operatorname{Prob}\{|\hat{N}-\langle\hat{n}\rangle| \geq \eta \sigma\}$ with $\eta \geq 0$. We write:

$$
Q(\eta)=\operatorname{Prob}\{|\hat{N}-\langle\hat{n}\rangle| \geq \eta \sigma\} \leq \Theta(\eta),
$$

with $\Theta(\eta)$ being a decreasing function of $\eta$. A rough estimate is given by the Chebyshev' inequality which implies: $\Theta(\eta)=\frac{1}{\eta^{2}}$.

We shall choose $N_{\text {inf }}$ such that:

$$
N_{\text {inf }} p_{H} \geq 1
$$

with a relatively high probability. Let us now formalize this condition by writing:

$$
N_{\text {inf }} p_{H}-\eta \sigma_{H}=N_{i n f} p_{H}-\eta \sqrt{N_{\text {inf }}} \sqrt{p_{H}\left(1-p_{H}\right)} \geq 1 .
$$


Eq.(25) expresses that, for a given $N_{i n f}$, the average number of components with corrects orientation exceeds unity by $\eta$ standard deviations. Solving Eq.(25), we obtain:

$$
N_{i n f} \geq \frac{\eta^{2}}{2 p_{H}}\left[\left(1-p_{H}\right)+\frac{2}{\eta^{2}}+\sqrt{\left(\left(1-p_{H}\right)+\frac{2}{\eta^{2}}\right)^{2}-\frac{4}{\eta^{4}}}\right] .
$$

Having determined $N_{i n f}$ we use Eq.(20) and the fact that $N(M, z) \leq$ $N_{\text {inf }}$ to derive the estimation:

$$
\langle M\rangle \geq\left\lceil\frac{\log \left[\frac{N_{i n f}}{N_{0}}\right]}{\log [\alpha(z)(1-p(z))]}\right\rceil,
$$

where the notation $\lceil x\rceil$ stands for the upper integer part of $x$.

Finally using Eq.(14), we can estimate the expected flow rate in the form:

$$
\mathcal{D}(\langle M\rangle, z)=\frac{\mathcal{R}(\langle M\rangle, z)}{u+\langle M\rangle\left(\tau_{0}+z\right)+\mathcal{R}(\langle M\rangle, z)(v+s)},
$$

where $\langle M\rangle$ is given by Eq.(27).

\subsection{Numerical illustration}

Let us give some numerical values for $N_{\text {inf }}$ obtained for $\eta=1$ and $\eta=2$. Eq.(26) implies:

$$
\eta=1 \Longrightarrow \begin{cases}N_{i n f} \geq 4+\sqrt{7} \approx 7 & \text { when } p_{H}=\frac{1}{3} \\ N_{\text {inf }} \geq 4 \text { when } p_{H}=\frac{1}{2} & \\ N_{\text {inf }} \geq \frac{7}{4}+\frac{\sqrt{13}}{3} \approx 3 & \text { when } p_{H}=\frac{2}{3}\end{cases}
$$

and similarly:

$$
\eta=2 \Longrightarrow \begin{cases}N_{i n f} \geq 7+2 \sqrt{10} \approx 13 & \text { when } p_{H}=\frac{1}{3} \\ N_{\text {inf }} \geq 4+2 \sqrt{3} \approx 8 & \text { when } p_{H}=\frac{1}{2} \\ N_{\text {inf }}=4.5 \approx 5 & \text { when } p_{H}=\frac{2}{3}\end{cases}
$$

In view of the above results the value $N_{i n f}=5$ offers a reasonable compromise for most applications.

Consider now the flow rates obtained with the following data obtained from the copper connector illustrated in Fig. 4.

$$
\begin{gathered}
z=1[\mathrm{sec}] \\
N_{0}=16[\mathrm{pce}]
\end{gathered}
$$




$$
\begin{gathered}
N_{\text {inf }}=5[\mathrm{pce}] \\
\lambda \simeq 0.12 \Rightarrow \alpha(z=1)=0.88 \\
p(z=2) \simeq p_{H}=0.55 \\
\tau_{0}=0.15[\mathrm{sec}] \\
u=0.5[\mathrm{sec}] \\
s=0.5\left[\frac{\mathrm{sec}}{\mathrm{pce}}\right] \\
v=0.3\left[\frac{\mathrm{sec}}{\mathrm{pce}}\right]
\end{gathered}
$$

With the these data, we obtain from Eq.(27):

$$
\langle M\rangle=2
$$

which leads from Eq.(18) with $M=\langle M\rangle=2$ to an average total yield $\mathcal{R}(\langle M\rangle, 2)=\mathcal{R}(2,2) \simeq 11$ [pce]. Not that this value is indeed consistent with $N_{0}=16$ and $N_{i n f}=5$. Finally from Eq.(28), the expected flow rate reads:

$$
\mathcal{D}(\langle M\rangle, 2)=\mathcal{D}(2,2) \simeq 0.93\left[\frac{\mathrm{pce}}{\mathrm{sec}}\right] \simeq 54\left[\frac{\mathrm{pce}}{\mathrm{min}}\right]
$$

Remark. The flow rate $\mathcal{D}(\langle M\rangle, z)$ will always be limited by the extraction time $s[\mathrm{sec}]$. Clearly $s=0.5[\mathrm{sec}]$ that was chosen above, is a lower bound. It is taken here to give an upperbound for the output of our module. More common values will be of the order of 2 [sec]. When this value is introduced, we have: $u+\langle M\rangle\left(\tau_{0}+z\right)<<(s+v) \mathcal{R}(\langle M\rangle, z)$. Hence, Eq.(18) can be approximated by:

$$
\mathcal{D}(\langle M\rangle, z)=\frac{\mathcal{R}(\langle M\rangle, z)}{u+\langle M\rangle\left(\tau_{0}+z\right)+\mathcal{R}(\langle M\rangle, z)(v+s)} \simeq \frac{1}{v+s},
$$

With the above data and $s=2[\mathrm{sec}]$, we have $\frac{1}{2.3}=0.43 \frac{[\mathrm{part}]}{[\mathrm{sec}]} \simeq$ $26 \frac{\text { part }]}{[\text { min] }}$. Directly using the complete expression Eq.(18), we obtain $\mathcal{D}(\langle M\rangle, z) \simeq 24 \frac{[\mathrm{part}}{[\mathrm{min}]}$.

\section{Conclusion}

Thank to the use of a vision system, a higher degree of flexibility can be achieved for parts feeding devices. Indeed, customized hardware such as: part orienting and selecting devices, separators, and linear conveyers can be suppressed and replaced by a vibrating tooling plate 
which combined in a single device the separation and the orientation of the components. Note that the increase of flexibility is done at the expense of a somehow complex feedback control of the device which fully takes into account the stochastic nature of the problem. This paper does also proposed a mathematical modeling of the sequence of operations. Beside a clarification of the operating modes, this abstract modeling enables to estimate the optimal set of external parameters relevant to operate the feeding device.

\section{References}

[Boo 82] G. Boothroyd, C. Poli and L. E. Murch. "Automatic Assembly." Marcel Dekker, (1982).

[Guck 83] J. Guckenheimer and Ph. Holmes. "Nonlinear Oscillations Dynamical Systems and Bifurcation of Vector Fields." Springer Verlag (1983). See in particular the section 2.4

[Hong 94] M.-O. Hongler "Chaotic and Stochastic Behavior in Automatic Production Lines." Lecture Note in Physics, Monograph m 22, Springer Verlag, (1994).

[Jaum 90] B. Jaumard, S.-H. Lu and C. Sriskandarajah. "Design Parameters and Ordering of Part Orienting Devices". Int. J. of Prod. Res. 28, (1990), 459-476.

[Lee 97] S. S. G. Lee, B. K. A. Ngoi, L. E. N. Lim and S. W. Lye. "Determining the probabilities of natural resting aspects of parts from their geometries" Assembly Automation 17, No 2, (1997), 137-142.

[Mey 75] S.L Meyer "Data Analysis for Scientists and Engineers." John Wiley, (1975)

[Red 67] A. H. Redford and G. Boothroyd "Vibrating feeding." Proc. Instn. Mech. Eng. 182, (1967-68), 135-146.

[Seth 90] S. P Sethi, C. Sriskandarajah and M. R. Rao. "Heuristic methods for selection and ordering of part orienting devices." Operation Res. 38, (1990), 84-98.

[Tuf 86] N. B. Tufillaro, T. M. Mello, Y. M. Choi and A.M. Albano. "Period doubling boundaries of a bouncing ball." J. Physique $\mathbf{4 7}$ (1986), 1477-1481.

[Wol 97] W. Wolfson and S. J. Gordon. "Designing a parts feeding system with maximum flexibility." Assmebly Aut. 17, No 2,(1997), 116-121. 